\title{
Excited state intramolecular charge transfer reaction in 4-(1-azetidinyl)benzonitrile: Solvent isotope effects
}

\author{
TUHIN PRADHAN, PIUE GHOSHAL and RANJIT BISWAS* \\ Department of Chemical, Biological and Macromolecular Sciences, and \\ Unit for Nano Science and Technology, S N Bose National Centre for Basic Sciences, JD Block, \\ Sector III, Salt Lake, Kolkata 700098 \\ e-mail: ranjit@bose.res.in
}

MS received 29 July 2008; revised 24 October 2008

\begin{abstract}
Excited state intramolecular charge transfer reaction of 4-(1-azetidinyl) benzonitrile (P4C) in deuterated and normal methanol, ethanol and acetonitrile has been studied in order to investigate the solvent isotope effects on reaction rates and yields. These quantities (reaction rates and yields) along with several other properties such as quantum yield and radiative rates have been found to be insensitive to the solvent isotope substitution in all these solvents. The origin of the solvent isotope insensitivity of the reaction is discussed and correlated with the observed slowing down of the solvation dynamics upon isotope substitution.
\end{abstract}

Keywords. TICT reaction; isotope effects; LE and CT emission decays.

\section{Introduction}

Isotope chemistry has been applied widely in the field of chemistry, from mechanistic studies of biochemical reactions to the determination of paleotemperatures from isotopic compositions of fossils. ${ }^{1-2}$ Deuterium substitution in solvents is known to modify both the static and dynamic nature of the solvents. For example, $\mathrm{D}_{2} \mathrm{O}$ is more structured and ordered liquid than $\mathrm{H}_{2} \mathrm{O}$ due to stronger $\mathrm{H}$-bond interactions in the deuterated water than in the normal species. In addition, the Debye relaxation times are slower in $\mathrm{D}_{2} \mathrm{O}$, whereas the peak frequencies of the intermolecular liberations and intramolecular vibrations lie at somewhat lower values than those in normal water. Solvation dynamics studies of isotope-substituted water ${ }^{3-4}$ and other polar solvents have revealed that the isotope-substitution affects the long time part of solvation energy relaxation which results into a slowing down of the solvation rate by $\sim 10-20 \%$. Because the dynamics of solvent reorganization is important for reactions where the solvent motion is coupled to the reaction coordinate, a slowing down in the solvent dynamics is expected to modify the reaction rate accordingly. Electron transfer (ET) or charge transfer (CT) reactions are

\footnotetext{
*For correspondence
}

such reactions where the solvation processes influence the reaction dynamics significantly.

Some studies have already used deuteriumsubstituted solute to investigate the effects of isotope on charge transfer reaction. ${ }^{5}$ Rotkiewicz et $a l^{6-7}$ have investigated the solute isotope effects on twisted intramolecular charge transfer (TICT) reactions by studying the fluorescence quantum yields, life times and the intersystem crossing yields. However, effects of isotopic substitution in solvents on reaction rates of intramolecular charge transfer reactions have not been investigated yet. In this article, we investigate the solvent isotope effects on the rate and yield of a photo-induced intramolecular charge transfer reaction in a molecule where the charge transfer in the photo-excited molecule is believed to occur simultaneously with the twisting of the donor group around the central bond that connects the acceptor moiety. The TICT molecule that we have used in the present study is 4-(1-azetidinyl) benzonitrile (P4C). Since the details of TICT mechanism has been discussed elsewhere, ${ }^{8-14}$ we refer to the schemes 1 and 2 in reference 8 to briefly discuss the reaction mechanism. As shown in these schemes, photo-excitation promotes P4C to the locally excited (LE) state in the first excited electronic surface $\left(S_{1}\right)$ which is relatively less polar and possesses charge distribution probably similar to that in the ground state. The 
photo-prepared LE state can then either undergo charge transfer intramolecularly (reaction rate $k_{f}$ ) or comes back to the ground $\left(\mathrm{S}_{0}\right)$ state with an average (radiative + non-radiative) rate, $k_{L E}$. Likewise, the charge transferred (CT) state can go back to the LE state with a rate of $k_{r}$ or use the radiative and nonradiative pathways with the average rate, $k_{C T}$ to populate the ground state. The activation barrier that separates the LE state from the more polar CT state is expected to be $\sim 6 k_{B} T^{12}$ where $k_{B} T$ denotes the Boltzmann's constant times the absolute temperature.

Since the TICT reactions have been found to couple partially to the medium dynamics, ${ }^{8-12}$ one naturally asks the following question: What could be the effects of the observed $\sim 10-20 \%$ slowing down of the long time solvation rate in deuterated solvents on the TICT reactions occurring in these solvents? More precisely, would the reaction rate in isotopically substituted solvents be slowed down accordingly? This is possible only when the twisting mode explores the full solvent redistribution time scale during the course of the TICT reaction. However, if the fast solvent reorganization at short times provides the necessary medium configuration (and thus the solvation energy), the reaction could become independent of solvent reorganization at long times. In such a scenario, the reaction rate is expected to be modified only by a very small amount. As the static dielectric constant $\left(\varepsilon_{0}\right)$ does not change significantly upon isotope substitution, the change in barrier height (solvent static effects) also remains minimal. Therefore, insignificant modifications in the both solvent static and dynamic effects upon isotope substitution would lead to the TICT reaction rate almost insensitive to the isotope labelling of the solvents.

The organization of the rest of the paper is as follows. Experimental details are given in the next section. Section 3 contains experimental results from our steady state and time resolved studies. The concluding remarks are in section 4 .

\section{Experimental details}

4-(1-azetidinyl) benzonitrile (P4C) was synthesized by following a protocol given in literature ${ }^{15}$ and recrystallized twice from cyclohexane (Merck, Germany). Purity of the compound was checked by thin layer chromatography and monitoring the excitation wavelength dependence of fluorescence emission in several bulk polar and non-polar solvents. Normal and deuterated methanol, ethanol and acetonitrile were used as received (spectrophotometric grade) from Aldrich. Steady state absorption spectra were recorded by using a spectrophotometer (Shimadzu, UV-2450). Steady state emission spectra were recorded by using a fluorimeter (SPEX fluoromax-3, Jobin-Yvon, Horiba) after adjusting the absorbance of the sample to $\sim 0 \cdot 1$. Measurements were made by taking the solutions in an optically transparent, quartz cuvette with $1 \mathrm{~cm}$ optical path length. For a given sample, the peak wavelength $(\lambda)$ of the absorption spectrum was used as excitation wavelength for the corresponding emission scan. The fluorescence spectra were corrected for the wavelength dependence of the sensitivity of the apparatus and properly converted to frequency representation for further analyses. Bubbling a few samples with dry argon gas showed very little or no effects on the final results. $^{8-12}$

The equilibrium constants $\left(K_{e q}\right)$ and the change in reaction free energy $\left(-\Delta G_{r}\right)$ for the $\mathrm{LE} \rightarrow \mathrm{CT}$ conversion reaction in $\mathrm{P} 4 \mathrm{C}$ were then determined from the area under the LE and CT bands obtained after deconvolution of the full emission spectrum of $\mathrm{P} 4 \mathrm{C}$ in a polar solvent into two fragments by using the reference emission spectrum of $\mathrm{P} 4 \mathrm{C}$ in perfluorohexane. ${ }^{12}$ Algebraic addition of the shifts of the emission spectra from the peak of the reference emission spectrum to the average peak frequency of the reference emission spectrum then provided the emission peak frequencies of the LE and CT bands. The average of the reference emission peak frequency was calculated by averaging the numbers obtained by fitting the upper half of the reference emission spectrum with an inverted parabola, first moment and the arithmetic mean of the frequencies at half intensities on both blue and red ends of the emission spectrum. ${ }^{16-18}$ Absorption peak frequencies were obtained by calculating the first moments of the absorption spectra. The error associated with the peak frequency determination is typically $\pm 250 \mathrm{~cm}^{-1}$ and that with the band area is $\sim 10 \%$ (of the reported value), unless otherwise mentioned.

Time resolved fluorescence emission intensity decays were collected using time correlated single photon counting (TCSPC) technique based on a laser system (Lifespec-ps, Edinburgh, UK) with a light emitting diode (LED) that provided $299 \mathrm{~nm}$ light as excitation. The full width at half maximum of the instrument response function (IRF) with the above excitation was approximately $475 \mathrm{ps}$. The emission 
decays were collected at magic angle at both LE and CT peak positions (of steady state spectrum) with an emission band pass of $8 \mathrm{~nm}$. Subsequently, the collected emission decays were deconvoluted from the IRF and fitted to multiexponential function using an iterative reconvolution algorithm. ${ }^{12}$ Such fitting enables one to capture decay kinetics with time constant as fast as $\sim 100 \mathrm{ps}$ with reasonable accuracy. ${ }^{12}$ For a few cases, emission decays were collected at two or three different emission wavelengths around the LE and CT peaks and the analysed data were found to vary within a small uncertainty. Note that while the emission (LE or CT) decays of P4C in these polar solvents (normal and deuterated) were found to be bi-exponential functions of time with one short and one long time constants, the decay in non-polar solvents (such as hexane or heptane) was single-exponential with only one long time constant. In addition, the short time constant (decay-time) obtained via unconstrained fit to the LE emission decay was found to be very similar to the short time constant (rise-time) required to fit the time dependent $\mathrm{CT}$ emission of $\mathrm{P} 4 \mathrm{C}$ in a given solvent. Therefore, the short time constant was considered as the reaction time for the $\mathrm{LE} \rightarrow \mathrm{CT}$ conversion reaction of $\mathrm{P} 4 \mathrm{C}$ in these polar solvents. All the experiments were performed at room temperature, $295 \pm 0.5 \mathrm{~K}$.

\section{Results and discussion}

As isotopic substitution induces no or insignificant changes in the polarity of the solvents considered here, the absorption and emission characteristics of a polarity probe in the deuterated solvents are not expected to differ much from those in the normal solvents. This is what is seen in the present study for all the solvents studied. Figure 1 shows the absorption and emission spectra of $\mathrm{P} 4 \mathrm{C}$ in ethanol and deuterated ethanol and the type of isotope insensitivity observed here is also seen for acetonitrile and methanol. Similar insensitivity has also been reported earlier by Lee et $a l^{19}$ with a non-reactive probe in normal and deuterated methanol and acetonitrile. The other spectral characteristics such as the absorption and emission peak frequencies $\left(v_{x}\right.$, $x=\mathrm{LE}$ or CT), spectral band-widths $(\Gamma)$ for both the LE and CT emission bands of P4C, and CT/LE area ratios $\left(\alpha_{\mathrm{CT}} / \alpha_{\mathrm{LE}}\right)$ in these solvents are summarized in table 1 . The CT/LE area ratio which is a measure of the reaction yield also remains insensitive as isotope substitution in solvent does not change the medium polarity $\left(\varepsilon_{0}\right)$. Note, however, that the area ratio $\left(\alpha_{\mathrm{CT}} /\right.$ $\left.\alpha_{\mathrm{LE}}\right)$ varies as the solvent is changed and the variation is roughly correlated with the direction in which the medium polarity increases. This is also reflected in the changes in the values of the equilibrium constant $\left(K_{e q}\right)$ and changes in reaction Gibbs free energy $\left(-\Delta G_{r}\right)$ for the $\mathrm{LE} \rightarrow \mathrm{CT}$ reaction of $\mathrm{P} 4 \mathrm{C}$. Calculations of these quantities $\left(K_{e q}\right.$ and $\left.-\Delta G_{r}\right)$ are described below.

The area ratio is related to the changes in reaction Gibbs free energy via the equilibrium constant in the following manner: ${ }^{8,12}$

$$
\Delta G_{r}=-\mathrm{RT} \ln K_{e q}=-\mathrm{RT} \ln \left(\alpha_{\mathrm{CT}} v_{\mathrm{LE}}^{3} / \alpha_{\mathrm{LE}} v_{\mathrm{CT}}^{3}\right) .
$$

Note that in table 1 the values of $-\Delta G_{r}$ and $K_{e q}$ of the reaction in deuterated methanol is slightly larger

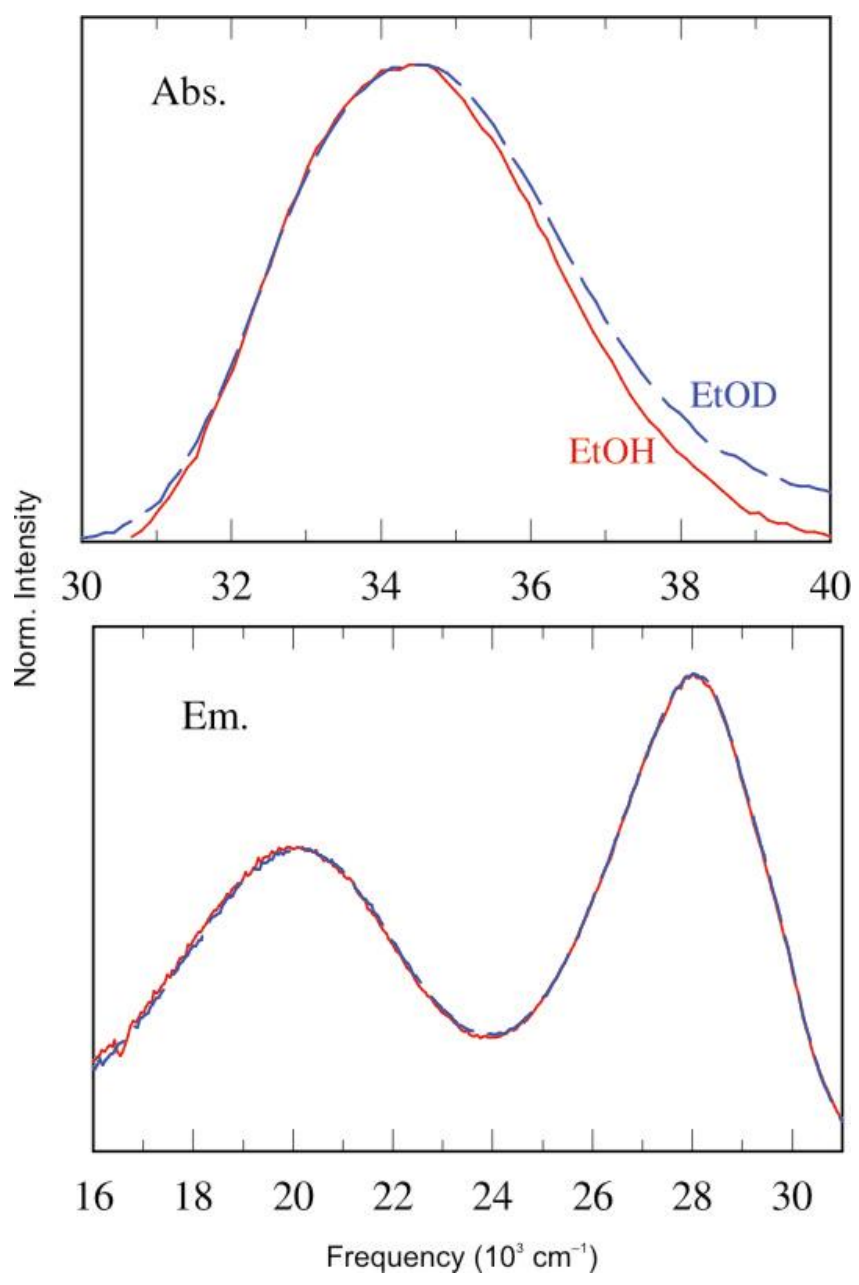

Figure 1. Absorption and emission spectra of 4-(1azetidinyl) benzonitrile (P4C) in deuterated and normal ethanol. Absorption spectra are shown in upper panel and emission spectra are shown in lower panel. Normal ethanol is represented by solid (red) and deuterated ethanol is represented by dashed (blue) lines. Similar effects of deuteration has also been found for acetonitrile and methanol. 
Table 1. Solvent isotope effects on spectral properties, area ratios, equilibrium constants of changes in reaction free energies. ${ }^{\text {a }}$

\begin{tabular}{|c|c|c|c|c|c|c|c|c|c|}
\hline Solvents & $v_{\mathrm{abs}}$ & $v_{\mathrm{LE}}$ & $v_{\mathrm{CT}}$ & $\Gamma_{\mathrm{abs}}$ & $\Gamma_{\mathrm{LE}}$ & $\Gamma_{\mathrm{CT}}$ & $\alpha_{\mathrm{CT}} / \alpha_{\mathrm{LE}}$ & $K_{\mathrm{eq}}$ & $-\Delta G_{r}$ \\
\hline $\mathrm{CH}_{3} \mathrm{OH}$ & 34.47 & $27 \cdot 76$ & $19 \cdot 60$ & $4 \cdot 42$ & 1.53 & $4 \cdot 18$ & $1 \cdot 35$ & $3 \cdot 85$ & $3 \cdot 34$ \\
\hline $\mathrm{CH}_{3} \mathrm{OD}$ & 34.47 & 27.77 & $19 \cdot 61$ & $4 \cdot 43$ & 1.53 & $4 \cdot 18$ & 1.42 & $4 \cdot 04$ & $3 \cdot 46$ \\
\hline $\mathrm{CH}_{3} \mathrm{CH}_{2} \mathrm{OH}$ & $34 \cdot 50$ & $27 \cdot 89$ & $20 \cdot 07$ & $4 \cdot 31$ & 1.42 & $4 \cdot 19$ & 0.93 & $2 \cdot 50$ & $2 \cdot 27$ \\
\hline $\mathrm{CH}_{3} \mathrm{CH}_{2} \mathrm{OD}$ & 34.69 & 27.90 & $20 \cdot 15$ & $4 \cdot 56$ & 1.42 & 4.09 & $0 \cdot 91$ & $2 \cdot 42$ & $2 \cdot 19$ \\
\hline $\mathrm{CH}_{3} \mathrm{CN}$ & 34.57 & $27 \cdot 44$ & $20 \cdot 05$ & $4 \cdot 32$ & $1 \cdot 33$ & $3 \cdot 72$ & 0.57 & 1.45 & 0.93 \\
\hline $\mathrm{CD}_{3} \mathrm{CN}$ & $34 \cdot 55$ & $27 \cdot 45$ & $20 \cdot 07$ & $4 \cdot 25$ & $1 \cdot 34$ & $3 \cdot 60$ & 0.56 & 1.44 & 0.91 \\
\hline
\end{tabular}

${ }^{\mathrm{a}}$ Peak frequencies $(v)$ and band widths $(\Gamma, \mathrm{fwhm})$ are in units of $10^{3} \mathrm{~cm}^{-1} . \alpha_{\mathrm{LE}} / \alpha_{\mathrm{CT}}$ denotes the area ratio between the $\mathrm{CT}$ and $\mathrm{LE}$ emission bands. $K_{e q}$ indicates the equilibrium constant for the $\mathrm{LE} \rightarrow \mathrm{CT}$ conversion reaction of $\mathrm{P} 4 \mathrm{C}$. Changes in reaction Gibbs free energy, $-\Delta G_{r}$ is in units of $\mathrm{kJ} /$ mole. Note that the error associated with peak frequency values is $\pm 250 \mathrm{~cm}^{-1}$ and that with band area is $\sim 10 \%$ of the reported value (a maximum of 4 measurements)

than those in normal methanol. Following the trend in dielectric relaxation parameters upon deuteration in water and also in the absence of dielectric relaxation data for these isotope-substituted solvents, we can only suggest that the small increase of these quantities is probably due to a slight increase in solvent polarity due to stronger H-bonds in deuterated methanol. However, deuteration of ethanol and acetonitrile probably does not change the solvent polarity $\left(\varepsilon_{0}\right)$ and therefore these quantities $\left(-\Delta G_{r}\right.$ and $\left.K_{e q}\right)$ remain the same as in the normal solvents. Dielectric relaxation studies of isotope-substituted methanol, ethanol and acetonitrile are therefore required to explain this insensitivity in a more quantitative manner.

Quantum yields $(\phi)$, radiative and non radiative rates $\left(k_{x}^{r a d}\right.$ and $\left.k_{x}^{n r}\right)$ associated with LE and CT bands have been calculated for $\mathrm{P} 4 \mathrm{C}$ in the normal and deuteratd solvents as follows: ${ }^{12}$

$$
\phi_{S}=\phi_{R}\left(\frac{n_{S}{ }^{2}}{n_{R}^{2}}\right)\left(\frac{I_{S}}{I_{R}}\right)\left(\frac{1-10^{-0.5 A_{R}}}{1-10^{-0.5 A_{S}}}\right) .
$$

Quinine sulphate dehydrates in $0.05 \mathrm{M} \mathrm{H}_{2} \mathrm{SO}_{4}$ has been used as reference $\left(\phi_{R}=0.508\right)$. In (2), n represents refractive index of the reference solution (R) and sample (S), and $I$ denotes the integrated emission intensity and $A$ the absorbance. Quantum yields of individual parts (LE and CT) for P4C molecule in deuterated and normal solvents have been determined and provided in table 2. Error associated with the determination of quantum yields is $\sim 20 \%$ of each of the reported values. Quantum yields of P4C in deuterated solvents are nearly the same as those in normal solvents. Values for radiative $\left(k^{\mathrm{rad}}\right)$, non radiative $\left(k^{n r}\right)$ and average life times $\left(\left\langle\tau_{\text {avg }}\right\rangle\right)$ can be determined by using our experimental data in the relations given in literature. ${ }^{12}$ For individual parts, however, we use the following relations: $k_{\mathrm{LB}}^{\mathrm{rad}}=\phi_{L E} /$ $\left\langle\tau_{L E}\right\rangle$, whereas average LE life time is obtained from the amplitudes $\left(a_{i}\right)$ and time constants $\left(\tau_{i}\right)$ obtained by fitting the relevant LE emission decays as follows: ${ }^{12}$

$$
\left\langle\tau_{\mathrm{LE}}\right\rangle=\sum_{i} a_{i} \tau_{i} / \sum_{i} a_{i} .
$$

The non-radiative rate for LE $\left(k_{L E}^{n r}\right)$ can then be calculated as follows: ${ }^{12} k_{L E}^{n r}=\left(1-\phi_{L E}\right) /\left\langle\tau_{L E}\right\rangle$. Similarly, $k_{C T}^{r a d}$ and $k_{C T}^{n r}$ can also be determined. These quantities for $\mathrm{P} 4 \mathrm{C}$ in the above solvent systems are calculated as discussed above and summarized in table 2 . It is evident from this table that isotope substitution in solvent either has very small or negligible effects on quantum yields and radiative rates. However, there seems to be a small decrease in non-radiative rates in isotope-substituted solvents and this is probably because of the relatively stronger structure of the deuterated solvents. The average LE and CT lifetimes also do not change (a maximum of $\sim 10 \%$ for CT life time in methanol) on isotope substitution of solvents. Interestingly, earlier studies ${ }^{6-7}$ with dimethyl aminobenzonitryl (DMABN) derivatives in deuterated water, methanol and ethanol reported much stronger isotope effects on fluorescence quantum yields and life times but no effects in acetonitrile. $^{7,20}$

Figure 2 shows the LE emission decays of P4C in deuterated (blue circles) and normal (red circles) ethanol where the bi-exponential fit parameters are also presented. The weighted residuals are also shown in the lower panels of this figure (figure 2) whose random fluctuations over time indicate the 
Table 2. Solvent isotope effects on quantum yields, radiative and non-radiative rates. ${ }^{\text {a }}$

\begin{tabular}{lccccccccc}
\hline Solvents & $\phi_{\text {net }}$ & $\phi_{\mathrm{LE}}$ & $\phi_{\mathrm{CT}}$ & $k_{\mathrm{LE}}^{\mathrm{rad}}$ & $k_{\mathrm{LE}}^{\mathrm{nr}}$ & $k_{\mathrm{CT}}^{\mathrm{rad}}$ & $k_{\mathrm{CT}}^{\mathrm{nr}}$ & $\left\langle\tau_{\mathrm{LE}}\right\rangle(\mathrm{ps})$ & $\left\langle\tau_{\mathrm{CT}}\right\rangle(\mathrm{ps})$ \\
\hline $\mathrm{CH}_{3} \mathrm{OH}$ & 0.017 & 0.007 & 0.010 & 5.11 & 7.02 & 0.85 & 0.87 & 141 & 1145 \\
$\mathrm{CH}_{3} \mathrm{OD}$ & 0.019 & 0.008 & 0.011 & 5.48 & 6.93 & 0.86 & 0.76 & 143 & 1299 \\
$\mathrm{CH}_{3} \mathrm{CH}_{2} \mathrm{OH}$ & 0.035 & 0.018 & 0.017 & 6.94 & 3.76 & 1.39 & 0.81 & 261 & 1210 \\
$\mathrm{CH}_{3} \mathrm{CH}_{2} \mathrm{OD}$ & 0.027 & 0.014 & 0.013 & $5 \cdot 24$ & 3.66 & 1.02 & 0.78 & 269 & 1264 \\
$\mathrm{CH}_{3} \mathrm{CN}$ & 0.04 & 0.026 & 0.014 & 4.71 & 1.80 & 0.93 & 0.63 & 542 & 1564 \\
$\mathrm{CD}_{3} \mathrm{CN}$ & 0.038 & 0.024 & 0.014 & 4.54 & 1.82 & 0.86 & 0.62 & 535 & 1589 \\
\hline
\end{tabular}

${ }^{\mathrm{a}} \phi_{\text {net }}$ indicates the net quantum yield associated with entire spectrum; $\phi_{\mathrm{LE}}$ and $\phi_{\mathrm{CT}}$ are the quantum yields of the individual parts (LE and CT); $k^{\text {rad }}$ (in units of $10^{7} \mathrm{~s}^{-1}$ ) and $k^{\mathrm{nr}}$ (in units of $10^{9} \mathrm{~s}^{-1}$ ) denote respectively the radiative and non-radiative rates corresponding to LE and CT bands. The errors associated with the net quantum yield and the average life times (LE and CT) are respectively $\sim 20 \%$ and $\sim 10 \%$ (of reported values), estimated from a maximum of four measurements

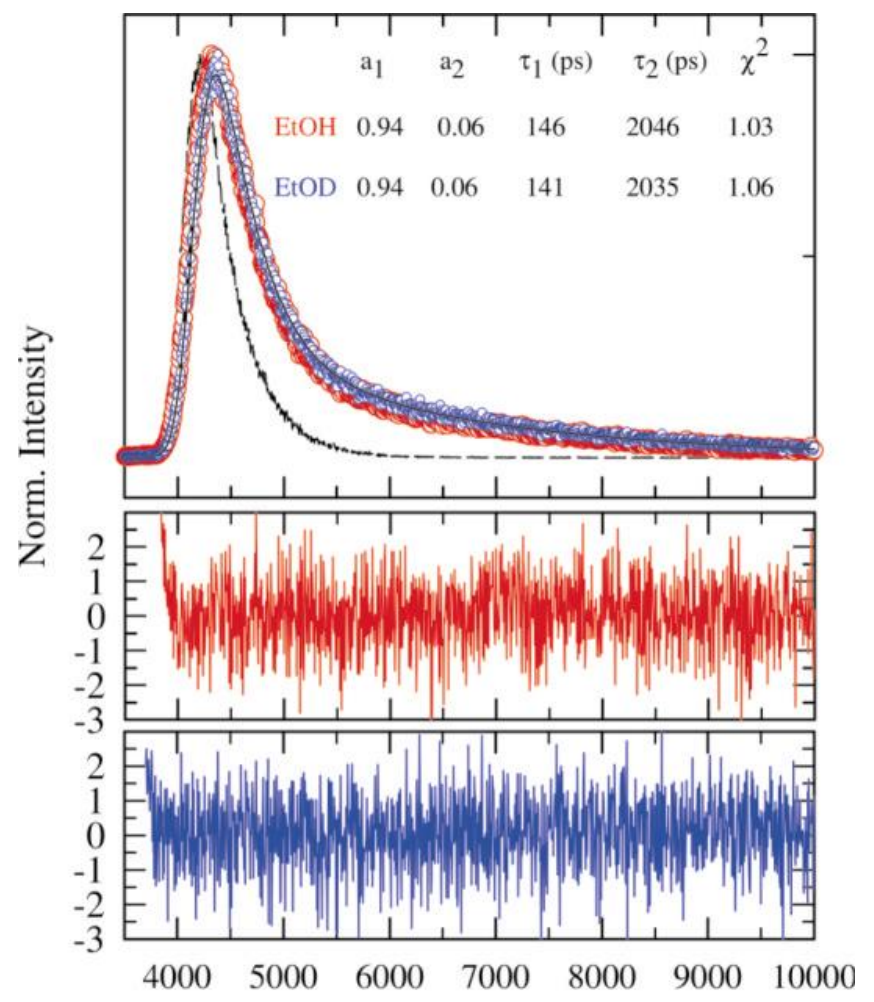

Time (ps)

Figure 2. Two representative LE emission decays of $\mathrm{P} 4 \mathrm{C}$ in deuterated (blue) and normal (red) ethanol. The data are represented by the circles, while the fit through the data are by the solid line. The instrument response function (IRF) is shown by the dashed line. The fit (biexponential) results are also provided in the inset of upper panel. The LE peak count is $\sim 3000$. Residuals are shown in the lower panel (colour code remains the same as in the upper panel).

adequacy of the bi-exponential function to fit the time dependent LE emission decays. We again men- tion here that the collected emission decays at wavelength near CT emission peaks could also be fitted with bi-exponential functions with same or similar time constants as found for the corresponding LE decays. For example, for P4C in methanol, fitting of CT emission decay with no constraints produces time constant 99 ps (rise time) and 1812 ps with $\chi^{2}=0.97$. This time constant (rise time) is thus very similar to the fast time constants (decay time, $96 \mathrm{ps}$ ) of the LE decay, indicating that these short time constants are essentially associated with the average reaction rate. It is evident from figure 2 as well as from the listed fit parameters that deuterium substitution in ethanol has negligible effects on the rate of the $\mathrm{LE} \rightarrow \mathrm{CT}$ reaction of $\mathrm{P} 4 \mathrm{C}$ in these solvents. The decay parameters obtained for deuterated and normal methanol and acetonitrile also show the similar comparison. The relevant data are summarized in table 3. As discussed earlier, ${ }^{9-11}$ short time constant associated with the LE decay is regarded as the average reaction time. The data in table 3 indicate that the isotope substitution neither changes the reaction time (short time constant) nor the amplitude of the associated component. This means that the reaction equilibrium ( $K_{e q} \alpha a_{\text {short }} / a_{\text {long }}$ ) is not changed upon isotope substitution, which is also a finding in the steady state studies (table 1). The long time constants in alcohol solvents, however, increases by $\sim 4-7 \%$ upon deuteration, and is again probably due to the better stabilization of the excited state in the more structured isotope-substituted alcohols studied here. Data in table 3 also indicate that deuterium substitution in acetonitrile has no effect on spectral properties as well as on the equilibrium constants and reaction kinetics. 
Table 3. Bi-exponential fit parameters for LE emission decays: Solvent isotope effects.

\begin{tabular}{lccccc}
\hline Solvent $^{\mathrm{a}}$ & $a_{1}$ & $a_{2}$ & $\tau_{1}(\mathrm{ps})$ & $\tau_{2}(\mathrm{ps})$ & $\chi^{2}$ \\
\hline $\mathrm{CH}_{3} \mathrm{OH}$ & 0.97 & 0.03 & 96 & 1804 & 0.98 \\
$\mathrm{CH}_{3} \mathrm{OD}$ & 0.97 & 0.03 & 94 & 1939 & 1.00 \\
$\mathrm{CH}_{3} \mathrm{CH}_{2} \mathrm{OH}$ & 0.94 & 0.06 & 146 & 2046 & 1.03 \\
$\mathrm{CH}_{3} \mathrm{CH}_{2} \mathrm{OD}$ & 0.94 & 0.06 & 141 & 2135 & 1.06 \\
$\mathrm{CH}_{3} \mathrm{CN}$ & 0.87 & 0.13 & 230 & 2579 & 0.95 \\
$\mathrm{CD}_{3} \mathrm{CN}$ & 0.87 & 0.13 & 230 & 2577 & 1.10 \\
\hline
\end{tabular}

${ }^{\mathrm{a}}$ The error associated with the amplitude estimates is $\sim 5 \%$ of the reported values

\section{Conclusion}

Solvent isotope effects on the reaction rate and yield of a twisted intramolecular charge transfer (TICT) reaction in deuterated methanol, ethanol and acetonitrile have been investigated in this article. Both the reaction rate and reaction yield have been found to have no effects from solvent isotope substitution. Other solute properties such as quantum yield, absorption and emission frequencies, line-widths and radiative rates also remain the same in deuterated solvents as those in the normal solvents. Nonradiative rates, however, appears to reduce by a few percent $(\leq 10 \%)$ in the alcohols studied here. The long time constant associated with the LE and CT decays of the TICT molecule (P4C) also lengthened by a small amount $(\sim 5 \%)$ on deuteration in methanol and ethanol, while deuteration in acetonitrile does not show any such effects. The average LE and CT lifetimes also show similar effects upon isotope substitution in solvents.

Even though the average solvation times in deuterated water and methanol have been found to be larger by $\sim 10 \%$, a proportionate slowing down of the TICT reaction rate in the deuterated solvents has not been observed here. Deuteration of solvents can affect a reaction in the following manner: (i) deuteration can slightly increase the static dielectric constant of a solvent which would reduce the reaction barrier involved in these reactions. This will enhance slightly the reaction rate (static solvent effects). (ii) The more structuredness in the deuterated solvents and less zero-point energy of $\mathrm{O}-\mathrm{D}$ bonds, on the other hand, would slow down the rate of the solvent orientational polarization relaxation. The relatively slow solvent polarization relaxation would then make the reactive mode (here the twisting mode) more coupled to the solvent dynamical modes producing a relatively stronger dynamical solvent control (dynamic solvent effects) of the reaction. Therefore, the insensitivity of the TICT reaction rates to the isotope substitution of these solvents may be a resultant of these two opposing solvent effects. This is probably the reason that even though the average solvation times in many of these solvents increases upon deuteration, the reaction rate remains insensitive. However, dielectric relaxation studies of these deuterated solvents are required to provide a more quantitative justification to the above discussion.

\section{Acknowledgements}

Financial supports from the Council of Scientific and Industrial Research (CSIR), India is gratefully acknowledged. One of the authors (TP) acknowledges the University Grant Commission (UGC), India for a research fellowship.

\section{References}

1. Iwase E, Tomioka A, Saigusa H and Yagi M 2004 Phys. Chem. Chem. Phys. 63852

2. Weston R E 1999 Chem. Rev. 992115

3. Nandi N, Roy S and Bagchi B 1995 J. Chem. Phys. 1021390

4. Zolotov B, Gan A, Fainberg B D and Huppert D 1997 J. Luminesce 72-74 842

5. Dresner J, Prochorow J and Sobolewski A 1978 Chem. Phys. Lett. 54292

6. Kohler G, Grabner G and Rotkiewicz K 1993 Chem. Phys. 173275

7. Grabowski Z R, Rotkiewicz K and Rettig W 2003 Chem. Rev. 1033899

8. Pradhan T and Biswas R 2007 J. Phys. Chem. A111 11514

9. Pradhan T and Biswas R 2007 J. Phys. Chem. A111 11524 
10. Pradhan T, Ghoshal P and Biswas R 2008 J. Phys. Chem. A112 915

11. Biswas R, Rohman N, Pradhan T and Buchner R 2008 J. Phys. Chem. A112 9379

12. Dahl K, Biswas R, Ito N and Maroncelli M $2005 \mathrm{~J}$. Phys. Chem. B109 1563

13. Gauduel Y, Pommeret S, Migus A and Antonetti A 1991 J. Phys. Chem. 95533

14. Gauduel Y, Pommeret S, Migus A and Antonetti A 1990 Chem. Phys. 1491
15. Rettig W 1980 J. Luminesc. 26 21; Rettig W 1982 J. Phys. Chem. 861970

16. Horng M L, Gardecki J A, Papazyan A and Maroncelli M 1995 J. Phys. Chem. B99 17311

17. Lewis J E, Biswas R, Robinson A G and Maroncelli M 2001 J. Phys. Chem. B105 3306

18. Lewis J E, Biswas R and Maroncelli M 1999 Chem. Phys. Lett. 310485

19. Lee S H, Lee J H and Joo T 1999 J. Chem. Phys. 11010969

20. Siebrand W 1971 J. Chem. Phys. 555843 